\title{
Passage Times for Unbiased Polymer Translocation through a Narrow Pore
}

\author{
Joanne Klein Wolterink, Gerard T. Barkema and Debabrata Panja \\ Institute for Theoretical Physics, Universiteit Utrecht, Minnaertgebouw, \\ Leuvenlaan 4, Postbus 80.195, 3508 TD Utrecht, The Netherlands
}

\begin{abstract}
We study the translocation process of a polymer in the absence of external fields for various pore diameters $b$ and membrane thickness $L$. The polymer performs Rouse and reptation dynamics. The mean translocation time $\left\langle\tau_{t}\right\rangle$ that the polymer needs to escape from a cell, and the mean dwell time $\left\langle\tau_{d}\right\rangle$ that the polymer spends in the pore during the translocation process, obey scaling relations in terms of the polymer length $N, L$ and $b / R_{g}$, where $R_{g}$ is the radius of gyration for the polymer. We explain these relations using simple arguments based on polymer dynamics and the equilibrium properties of polymers.
\end{abstract}

PACS numbers: 36.20.-r, 82.35.Lr, 87.15.Aa

Transport of molecules across membranes is an essential mechanism for life processes. These molecules are often long, and the narrow pores in the membranes do not allow them to pass through as a single unit. They have to thus squeeze - i.e., translocate - themselves through the pores. DNA, RNA and proteins are such naturally occuring long molecules [1, 2, 3, 4, 5] in a variety of biological processes. Translocation is used in gene therapy [6, 7], and in delivery of drug molecules to their activation sites $[8,9]$. Understandably, the process of translocation has been an active topic of current research: not only because it is a cornerstone of many biological processes, but also due to its relevance for practical applications.

Translocation is a complicated process in living organisms - the presence of chaperon molecules, $\mathrm{pH}$, chemical potential gradients, and assisting molecular motors strongly influence its dynamics. Consequently, the translocation process has been empirically studied in great variety in biological literature [10, 11]. Study of translocation as a biophysical process is more recent. Herein, the polymer is simplified to a sequentially connected string of $N$ monomers as it passes through a narrow pore on a membrane. The quantities of interest are the typical time scale for the polymer to leave a confining cell (the "escape of a polymer from a vesicle" time scale) 12], and the typical time scale the polymer spends in the pore (the "dwell" time scale) [13] as a function of $N$ and other parameters like membrane thickness, membrane adsorption, electrochemical potential gradient, etc. 14].

These quantities have been measured directly in numerous experiments [15]. A number of (mean-field type) theories have been proposed for the scaling of these typical times [12, 13, 14] during the last decade as well. They describe translocation as a first-passage or Kramer's problem over an entropic barrier in terms of the "reaction coordinate" $m$ alone. Here $m$ is the number of the monomer threaded into the pore $(m=1, \ldots, N)$, and the transition rates from $m$ to $m \pm 1$ and vice versa are obtained from the derivatives of the free energy w.r.t. $m$.

In the context of unbiased polymer translocation (i.e., in the absence of external driving fields), the prediction of the mean-field theories (which only consider polymers with simple random walk statistics) is that the dwell time scales as $N^{3}$ for Rouse dynamics and as $N^{2.5}$ for Zimm dynamics [13]. These theories indeed provide insight into the process of translocation, but their usage of the (equilibrium) free energy to determine the transition rates from $m$ to $m \pm 1$ implicitly assumes that at a fixed reaction coordinate $m$ the polymers equilibriate much faster than the typical time for the reaction coordinate to change its value by \pm 1 . This is not necessarily the case for longer polymers or polymers in higher spatial dimensions 16. Verifying the scaling results of these theories using simulations, too, remains a computationally significant challenge since it involves simulating long polymers and correspondingly long time scales.

The purpose of this letter is (a) to report the results of extensive lattice-based Monte Carlo simulations of the unbiased translocation process in three spatial dimensions, for a variety of polymer lengths, pore diameters and membrane thickness; and (b) to trace the physical origin of their differences from the existing mean-field theory results. Our system consists of two cells A and $\mathrm{B}$, each of volume $V$, that are connected by a pore of diameter $b$ in a membrane of thickness $L$. The polymer is modeled as a lattice polymer of $N$ monomers, obeying self-avoiding walk statistics. Its movement consists of single monomer jumps to neighboring lattice sites. Jumps along the contour of the polymer, i.e., reptation moves, are attempted with a higher frequency than jumps that displace the contour of the polymer laterally to cause Rouse dynamics. A detailed description of this lattice polymer model, its computationally efficient implementation and a study of some of its properties and applications can be found in Ref. 17, 18]. Hydrodynamical interactions are not incorporated in this model.

In our simulations, the polymer repeatedly moves back and forth from one cell to the other through the pore [see Fig. 1(a)]. Our primary interest lies in the scaling behaviour of two quantities, (i) the mean translocation time $\left\langle\tau_{t}\right\rangle$, the time required for the whole polymer to escape from one cell to the other, and (ii) the mean dwell time $\left\langle\tau_{d}\right\rangle$, the time that the polymer spends in the pore during the translocation process. To define these quantities precisely, we introduce the following states of the polymer. In states $\mathrm{A}$ and $\mathrm{B}$, the entire polymer is lo- 


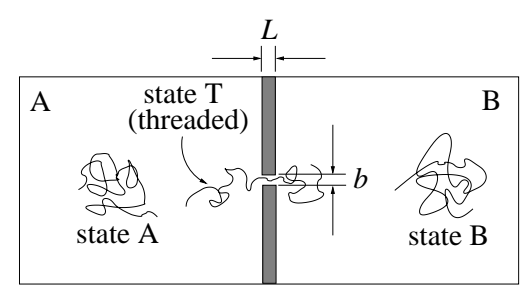

(a)

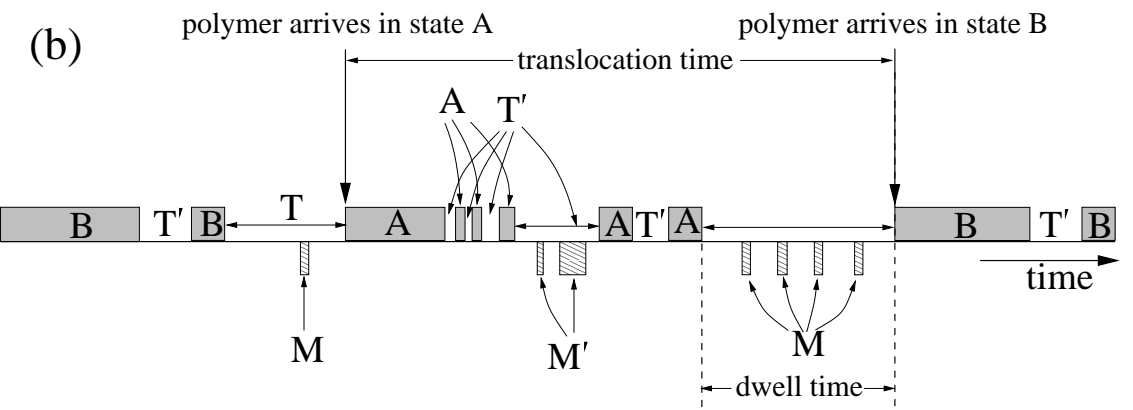

FIG. 1: (a) Our system with polymers in different states. (i) state A: all monomers are in cell A; (ii) state T (threaded): some monomers are in cell A and the rest in cell B; (iii) state B: all monomers are in cell B. (b) A typical translocation process of the polymer [using the definition of polymer states defined in Fig. 11a)] for our system.

cated in cell A, resp. B. States $\mathrm{M}$ and $\mathrm{M}^{\prime}$ are defined as the states in which the middle monomer is located halfway between both cells. Finally, states $\mathrm{T}$ and $\mathrm{T}^{\prime}$ are the complementary to the previous states: the polymer is threaded, but the middle monomer is not in the middle of the pore. The finer distinction between states $\mathrm{M}$ and $\mathrm{T}$, resp. $\mathrm{M}^{\prime}$ and $\mathrm{T}^{\prime}$ is that in the first case, the polymer is on its way from state $A$ to $B$ or vice versa, while in the second case it originates in state A or B and returns to the same state. The translocation process in our simulations can then be characterized by the sequence of these states in time (see Fig. (1). In this picture, the dwell time is the mean time that the polymer spends in states $\mathrm{M}$ or $\mathrm{T}$, while the translocation time is the mean time starting at the first instant the polymer reaches state $\mathrm{A}$ after leaving state B, until it reaches state B again.

Having set both Kuhn length of the polymer and the lattice spacing to unity, we conjecture that for thin membranes $(L \simeq 1)\left\langle\tau_{d}\right\rangle \sim N^{1+2 \nu} F\left(b / R_{g}\right)$, and verify it for $b=1$ using polymer lengths up to $N=$ 1200. For narrow pores $(b \simeq 1)$, we argue and verify that $\log \left\langle\tau_{t}\right\rangle \sim L$. We also observe that $\left\langle\tau_{t}\right\rangle \sim$ $V N\left(b / R_{g}\right)^{-\left(1+2 \nu+\gamma-2 \gamma_{1}\right) / \nu} F\left(b / R_{g}\right)$. Here $\nu=0.588$ is the growth exponent for self-avoiding walks, and $\gamma=$ 1.1601 and $\gamma_{1}=0.68$ are exponents related to the entropy of a polymer in bulk and near a rigid wall respectively, and $F(\xi)$ is a scaling function; it approaches a constant for $\xi \rightarrow \infty$ and behaves $\sim \xi^{-0.38 \pm 0.08}$ as $\xi \rightarrow 0$.

Argument for the scaling of $\left\langle\tau_{d}\right\rangle$ : For thin membranes $(L \simeq 1)$, a scaling relation between $\left\langle\tau_{d}\right\rangle, b$ and $N$ can be obtained by the following hypothesis: $\left\langle\tau_{d}\right\rangle=$ $N^{\alpha} F\left(b / N^{\beta}\right)$ for some $\alpha$ and $\beta$. We expect that for $\left\langle\tau_{d}\right\rangle, b / R_{g}$ is a relevant dimensionless parameter that determines how easily the polymer can squeeze itself through the pore, since the polymer can "feel" the presence of the pore only if its radius of gyration $R_{g}$ is comparable to the pore diameter $b$. This implies that $b / N^{\beta} \sim b / R_{g} \Rightarrow \beta=\nu$, as $R_{g}=\lambda N^{\nu}$. Moreover, from physical grounds, such a scaling hypothesis means that the scaling function $F(\xi)$ should approach a constant for $\xi \rightarrow \infty$. Since for very large pores (i.e., $b \gg R_{g}$ ) the polymer no longer feels the pore, $\left\langle\tau_{d}\right\rangle$ should be the time taken by the polymer to diffuse a distance $R_{g} \Rightarrow\left\langle\tau_{d}\right\rangle \sim R_{g}^{2} /\left(2 D_{N}\right) \sim R_{g}^{2} N \sim N^{1+2 \nu}$, where $D_{N} \sim N^{-1}$ is the diffusion coefficient of the polymer in a dilute polymer solution. The last relation, together with the scaling hypothesis implies that $\alpha=1+2 \nu$, and

$$
\left\langle\tau_{d}\right\rangle \sim N^{2 \nu+1} F\left(\frac{b}{R_{g}}\right) .
$$

Additionally, since the monomers within the pore move along the contour of the polymer, i.e., reptate, $\left\langle\tau_{d}\right\rangle$ should be independent of $L$, as long as $L \ll N$ [19].

Relation between $\left\langle\tau_{d}\right\rangle$ and $\left\langle\tau_{u}\right\rangle$ : During the dwelling process, the polymer necessarily has to pass state $\mathrm{M}$ at least once. Due to the spatial symmetry between cells A and B, each time sequence as depicted in Fig. 11 is equally probable under exchange of states A and B. Additionally, each time sequence from A to B is as likely as its timereversed counterpart.

To devise a computationally cheaper method to measure $\left\langle\tau_{d}\right\rangle$ using these symmetries, we introduce an additional time-scale $\left\langle\tau_{u}\right\rangle$, the mean unthreading time, which is the average time that either state $\mathrm{A}$ or $\mathrm{B}$ is reached from state $M$ (not excluding possible reoccurrences of state M). Due to time symmetry, the mean time passed since the polymer last left state A, until it reached state $\mathrm{M}$, is as large as the mean time passed since the polymer last left state B, until it reached state M. Consequently,

$$
\left\langle\tau_{d}\right\rangle=2\left\langle\tau_{u}\right\rangle .
$$

For $b>1$, we expect a similar relation between $\left\langle\tau_{d}\right\rangle$ and $\left\langle\tau_{u}\right\rangle$ to hold. However, we do not have a suitable argument for it, since for larger pores, the properties of the polymer as a macromolecule start to play a role [20].

Relation between $\left\langle\tau_{t}\right\rangle$ and $\left\langle\tau_{d}\right\rangle$ for $b=1$ : The fraction of time spent in states $\mathrm{M}$ and $\mathrm{T}$ compared to $\left\langle\tau_{t}\right\rangle$ equals $\left\langle\tau_{d}\right\rangle /\left\langle\tau_{t}\right\rangle$. The probability that the polymer is threaded exactly halfway is an equilibrium property; hence, the sum of the probabilities $p_{\mathrm{M}}$ and $p_{\mathrm{M}}^{\prime}$ that the polymer is in state $\mathrm{M}$ or $\mathrm{M}^{\prime}$ can be obtained from the contribution 
of these states to the total partition sum $Z_{\mathrm{tot}}$. The ratios $f_{\mathrm{M}} \equiv p_{\mathrm{M}} / p_{\mathrm{M}^{\prime}}$ and $f_{\mathrm{T}} \equiv p_{\mathrm{M}} / p_{\mathrm{T}}$ are non-equilibrium properties, but as we show below, it is possible to estimate these ratios accurately from targeted simulations. With these quantities, the average translocation time can be obtained indirectly from the dwell time, using

$$
\left\langle\tau_{t}\right\rangle=\left\langle\tau_{d}\right\rangle \frac{f_{\mathrm{T}}\left(1+f_{\mathrm{M}}\right)}{\left(p_{\mathrm{M}}+p_{\mathrm{M}^{\prime}}\right) f_{\mathrm{M}}\left(1+f_{\mathrm{T}}\right)} .
$$

We verify Eqs. (13) and cross-check their consistency using direct simulations to measure $\left\langle\tau_{t}\right\rangle$ and targeted simulations to measure $\left\langle\tau_{u}\right\rangle, p_{\mathrm{M}}+p_{\mathrm{M}^{\prime}}, f_{\mathrm{M}}$ and $f_{\mathrm{T}}$. We use the lattice polymer model of Ref. [17].

First, we estimate the entropic penalty paid by the polymer in state $\mathrm{M}$ or $\mathrm{M}^{\prime}$. The partition sum $Z_{b}(N)$ of a self-avoiding polymer of length $N$, anchored at the origin of an infinite lattice, is proportional to $Z_{b}(N) \sim$ $\mu^{N} N^{\gamma-1}$, with $\gamma=1.1601$ and $\mu$ is a lattice-dependent non-universal constant. If this polymer is restricted to the half-space $z \geq 0$, the same expression holds, but with an adjusted exponent $\gamma_{1}=0.68$ 21]. The partition sum of a polymer of length $N$ in state $\mathrm{M}$ or $\mathrm{M}^{\prime}$, threaded through a narrow pore $(b=1)$ in a thin membrane $(L=1)$ is then given by the product of the partition functions of two separate self-avoiding polymers of length $N / 2$, each having one of their ends anchored at a rigid wall, as $Z_{t}(L=1, N / 2, N / 2)=\left[\mu^{N / 2}(N / 2)^{\gamma_{1}-1}\right]^{2} \sim$ $Z_{b}(N) N^{-\gamma+2 \gamma_{1}-1}$. Adding a linear scaling with $V$ to $Z_{b}(N)$, this ratio is also the equilibrium probability that the polymer is in state $\mathrm{M}$ or $\mathrm{M}^{\prime}$. Hence, we obtain

$$
p_{\mathrm{M}}+p_{\mathrm{M}^{\prime}}(N) \sim N^{-\gamma+2 \gamma_{1}-1} / V .
$$

With increasing membrane thickness $L$, since the lattice coordination number in the pore is much smaller than in the bulk, we have $\log Z_{t}(L, N) \sim L$, at least as long as $L \ll N$ (neglecting logarithmic terms). Stated differently, the entropic barrier encountered by the translocating polymer increases linearly with $L$. This results in an exponential increase for $\left\langle\tau_{t}\right\rangle$ [see Fig. 3]:

$$
\log \left\langle\tau_{t}\right\rangle \sim L
$$

Next, we perform dynamical simulations to determine $f_{\mathrm{M}}, f_{\mathrm{T}}$ and $\left\langle\tau_{u}\right\rangle$. The simulations start with a polymer of length $N$, threaded halfway in the pore, and the polymer originates from cell $\mathrm{A}$. We then wait until the polymer unthreads. If it unthreads into cell $\mathrm{A}$, the starting configuration is labeled $\mathrm{M}^{\prime}$, while if it unthreads into cell $\mathrm{B}$, it is labeled $\mathrm{M}$. We record the ratio of the number of polymers unthreading into cell A vs. cell B for polymer lengths $N=20,40,50$ and 80 , and obtained 1.37, 1.28, 1.33 and 1.27 respectively for this ratio; i.e., cell $\mathrm{A}$ is preferred above cell B by a small factor, which does not increase with $N$. This asymmetry can be easily explained by the fact that during the translocation process from state A to state B, the polymer accumulates folded segments on the B side and stretched segments on the A side, which makes it more prone to go back to state A than to proceed to state B. Thus, with $c \simeq 1.3^{-1}$, we conclude that

$$
f_{\mathrm{M}}(N) \sim c .
$$

In the same simulations, we measured $f_{\mathrm{T}} /\left(1+f_{\mathrm{T}}\right)$, i.e., the fraction of time that the polymer is in state $\mathrm{M}$ before unthreading. From the theoretical ratio of $\sum_{i=1}^{N-1} Z_{t}(L=1, i, N-i)$ and $Z_{t}(L=1, N / 2, N / 2)$, we found (and numerically observed as well) that

$$
f_{T}(N) \sim N^{-1}
$$

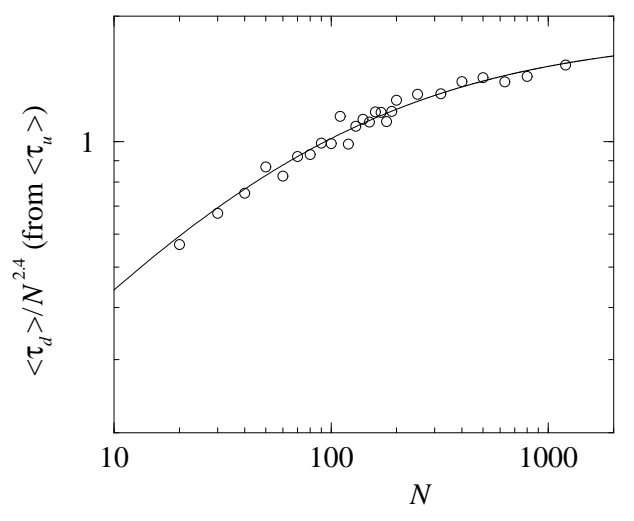

FIG. 2: $\left\langle\tau_{d}\right\rangle$ obtained from $\left\langle\tau_{u}\right\rangle$ for $L=b=1$ and $N$ up to 1200. Solid line: $\left\langle\tau_{d}\right\rangle=\left[0.55 N^{-3}+6.84 N^{-2.4}\right]^{-1}$. When the same data are plotted in the $\left(b / R_{g}\right)-\left(\left\langle\tau_{d}\right\rangle / N^{1+2 \nu}\right)$ coordinates, the $\xi^{-0.38 \pm 0.08}$ scaling for $F(\xi)$ is recovered for $\xi \rightarrow 0$.

For each $N$, we combined the unthreading times into a histogram. We then obtained $\left\langle\tau_{u}\right\rangle(N)$ from a fit of the long-times tail of this histogram (see Fig. 2). We found that for short polymers, $\left\langle\tau_{u}\right\rangle(N) \sim N^{3}$, while for long polymers, $\left\langle\tau_{u}\right\rangle(N) \sim N^{2.4 \pm 0.05}$. The explanation of this is as follows: a polymer translocating from A to B pulls on its segments in A as it accumulates folded segments in B. The resulting strains can be released by reptation moves (along the contour) that initiate only at the ends, or by Rouse moves (perpendicular to the contour) that take place anywhere on the polymer. For the first mechanism, the scaling of $\left\langle\tau_{u}\right\rangle$ with $N$ is that of reptation [i.e., $\left\langle\tau_{u}\right\rangle(N) \sim N^{3}$, which is the same as the mean-field theory result], but since there are of $O(N)$ more segments on the polymer (where Rouse moves can occur) than the two ends (where reptation moves initiate), the second one dominates for long polymers, giving rise to the crossover seen in Fig. 2 The precise location of this crossover depends on the details of the experiment/simulation.

Having combined Eqs. (24) and (6 7), we obtain, for $b=1,\left\langle\tau_{t}\right\rangle$ scaling as a function of polymer length as

$$
\left\langle\tau_{t}\right\rangle \sim V N^{2+2 \nu+\gamma-2 \gamma_{1}+0.22 \pm 0.05}
$$


For $b>1$ and $L=1$, a scaling relation for $\left\langle\tau_{t}\right\rangle$ can be obtained if we assume that $\left\langle\tau_{t}\right\rangle$ is related to $\left\langle\tau_{d}\right\rangle$ in the same way as in Eq. (3). In this case we expect the entropic penalty paid by the polymer in state $\mathrm{M}$ or $\mathrm{M}^{\prime}$ to behave as a function of $b / R_{g}$ - as explained above Eq. (11), this is the quantity that determines to what extent the polymer "feels" the presence of the pore, i.e., we expect $N^{-\gamma+2 \gamma_{1}-1}$ in Eq. (4) to be replaced by $\left(N^{\nu} / b\right)^{\left(-\gamma+2 \gamma_{1}-1\right) / \nu}$. Simultaneously, $V$ in Eq. (44) is to be replaced by $V / b^{2}$, as the chance for the polymer to find the pore increases linearly with the pore area. We however expect Eqs. (6) and (7) to remain unchanged as they only concern a threaded polymer. Together with Eq. (1), for $b>1$ and $L=1$ this argument leads one to

$$
\left\langle\tau_{t}\right\rangle \sim V N\left(b / R_{g}\right)^{-\left(1+2 \nu+\gamma-2 \gamma_{1}\right) / \nu} F\left(b / R_{g}\right) .
$$

We performed direct simulations to verify Eq. (9) [see Fig. 3. We started with a polymer in cell A, as shown in Fig. 1 and recorded the times (for up to 500 different runs) it took to reach state B. We then made a histogram of these times and deduced $\left\langle\tau_{t}\right\rangle$ from its asymptotic slope.

In summary, we studied unbiased polymer translocation for various pore diameters $b$ and membrane thicknesses $L$, using a lattice polymer model. We found that for $L=1$, both the mean translocation time $\left\langle\tau_{t}\right\rangle$ and the mean dwell time $\left\langle\tau_{d}\right\rangle$ obey scaling relations that involve functions of $b / R_{g}$, where $R_{g}$ is the radius of gyration of the polymer. We also showed that for $b=1, \ln \left\langle\tau_{t}\right\rangle \sim L$ and $\left\langle\tau_{d}\right\rangle$ is independent of $L$. We explained these results using simple arguments based on the polymer's dynamical and equilibrium properties. Our analysis explains, for the first time, how and why deviations from the mean- field theory results occur for long polymers.

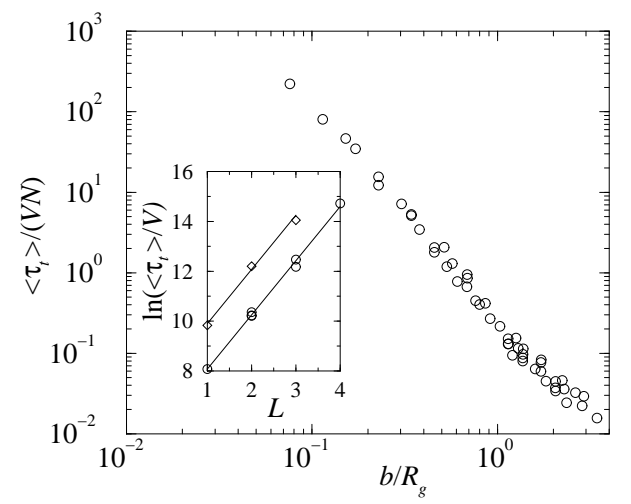

FIG. 3 : $\left\langle\tau_{t}\right\rangle$ vs. $b / R_{g}$ for $N=20,40,80 ; b=1, \ldots, 31$. Inset: $\ln \left\langle\tau_{t}\right\rangle \sim L$ for $b=1$ (circles: $N=20$, diamonds: $N=40$ ).

The persistence length of a polymer in translocation experiments is equivalent to the Kuhn length used here. In experiments electric field effects due to the applied bias voltage and hydrodynamical effects 22] are always present; these we did not consider here. Nevertheless, as our scaling results are based on very general grounds, we expect the same scaling forms (involving $b / R_{g}$ for $L=1$, or $\ln \left\langle\tau_{t}\right\rangle \sim L$ for $b=1$ ) to hold when the hydrodynamical effects are taken into account, albeit with different exponents. The effects of hydrodynamics and external fields on translocation are two major topics of our onging work.

We thank Profs. Henk van Beijeren and Erich Eisenriegler for helpful discussions.
[1] B. Dreiseikelmann, Microbiol. Rev. 58, 293 (1994).

[2] J. P. Henry et al., J. Membr. Biol. 112, 139 (1989).

[3] J. Akimaru et al., PNAS USA 88, 6545 (1991).

[4] D. Goerlich and T. A. Rappaport, Cell 75, 615 (1993).

[5] G. Schatz and B. Dobberstein, Science 271, 1519 (1996).

[6] I. Szabò et al. J. Biol. Chem. 272, 25275 (1997).

[7] B. Hanss et al., PNAS USA 95, 1921 (1998).

[8] Yun-Long Tseng et al., Molecular Pharm. 62, 864 (2002).

[9] J. M. Tsutsui et al., Cardiovasc. Ultrasound 2, 23 (2004).

[10] W. T. Wickner and H. F. Lodisch, Science 230, 400 (1995).

[11] S. M. Simon and G. Blobel, Cell 65, 1 (1991); D. Goerlich and I. W. Mattaj, Science 271, 1513 (1996); K. Verner and G. Schatz, Science 241, 1307 (1988).

[12] P. J. Sung and W. Park, Phys. Rev. E 57, 730 (1998); M. Muthukumar, Phys. Rev. Lett. 86, 3188 (2001).

[13] P. J. Sung and W. Park, Phys. Rev. Lett. 77, 783 (1996); M. Muthukumar, J. Chem. Phys. 111, 10371 (1999).

[14] D. K. Lubensky and D. R. Nelson, Biophys. J. 77, 1824 (1999); P. J. Park and W. Sung, J. Chem. Phys. 108, 3013 (1998); E. Slonkina and A. B. Kolomeisky, J. Chem. Phys. 118, 7112 (2003).

[15] J. Kasianowicz et al., PNAS USA 93, 13770 (1996); E.
Henrickson et al., Phys. Rev. Lett. 85, 3057 (2000); A Meller et al., Phys. Rev. Lett. 86, 3435 (2001); M. Akeson et al., Biophys. J. 77, 3227 (1999); A. Meller et al., PNAS USA 97, 1079 (2000); A. J. Storm et al., Nanoletters 5, 1193 (2005).

[16] J. Chuang et al., Phys. Rev. E 65, 011802 (2002); Y. Kantor and M. Kardar, Phys. Rev. E 69, 021806 (2004).

[17] A. van Heukelum and G. T. Barkema, J. Chem. Phys. 119, 8197 (2003).

[18] A. van Heukelum et al., Macromol. 36, 6662 (2003); J. Klein Wolterink et al., Macromol. 38, 2009 (2005); J. Klein Wolterink and G. T. Barkema, Mol. Phys. 103, 3083 (2005).

[19] Note that Eq. (1) without the scaling function $F\left(b / R_{g}\right)$ was obtained in Ref. [16] in two dimensions, for $N \simeq 100$.

[20] For $b>1$, in state $\mathrm{M}$ or $\mathrm{M}^{\prime}$, the two ends of the polymer may end up in the same cell. How to account for such states is not clear to us.

[21] H. W. Diehla and M. Shpot, Nucl. Phys. B 528, 595 (1998).

[22] In translocation experiments, pore blockage can be detected with short AC (instead of DC) pulses; this eliminates the effect of the external fields. 